\title{
Ultra-Sensitive Flexible Tactile Sensor Based on Graphene Film
}

\author{
Xiaozhou Lü ${ }^{1, * \mathbb{C}}$, Liang $\mathrm{Qi}^{1}{ }^{1}$, Hanlun $\mathrm{Hu}^{1}{ }^{1}$, Xiaoping Li ${ }^{1}$, Guanghui Bai ${ }^{2}$, Jun Chen ${ }^{3}$ \\ and Weimin Bao ${ }^{1}$ \\ 1 School of Aerospace Science and Technology, Xidian University, Xi'an 710071, China; \\ lqi_1@stu.xidian.edu.cn (L.Q.); huhanluna@163.com (H.H.); xpli@xidian.edu.cn (X.L.); \\ baoweimin@cashq.ac.cn (W.B.) \\ 2 Science and Technology on Space Physics Laboratory, Beijing 100076, China; baigh_2019@163.com \\ 3 China Academy of Launch Vehicle Technology, Beijing 100076, China; bghbuaa@aliyun.com \\ * Correspondence: 1xz@uw.edu
}

Received: 22 September 2019; Accepted: 22 October 2019; Published: 28 October 2019

\begin{abstract}
Flexible tactile sensor can be integrated into artificial skin and applied in industrial robot and biomedical engineering. However, the presented tactile sensors still have challenge in increasing sensitivity to expand the sensor's application. Aiming at this problem, this paper presents an ultra-sensitive flexible tactile sensor. The sensor is based on piezoresistive effect of graphene film and is composed of upper substrate (PDMS bump with a size of $5 \mathrm{~mm} \times 7 \mathrm{~mm}$ and a thickness of $1 \mathrm{~mm}$ ), medial Graphene/PET film (Graphene/PET film with a size of $5 \mathrm{~mm} \times 7 \mathrm{~mm}$, PET with a hardness of $2 \mathrm{H}$ ) and lower substrate (PI with fabricated electrodes). We presented the structure and reduced the principle of the sensor. We also fabricated several sample devices of the sensor and carried out experiment to test the performance. The results show that the sensor performed an ultra high sensitivity of $10.80 \Omega / \mathrm{kPa}$ at the range of $0-4 \mathrm{kPa}$ and have a large measurement range up to $600 \mathrm{kPa}$. The sensor has 4 orders of magnitude between minimum resolution and maximum measurement range which have great advantage compared with state of the art. The sensor is expected to have great application prospect in robot and biomedical.
\end{abstract}

Keywords: flexible tactile sensors; ultra-sensitive; graphene film

\section{Introduction}

Flexible tactile sensor is an electronic device which is able to mimics human skin tactile receptors. It can be integrated into artificial skin to get pressure information from external stimulation. With the development of artificial skin, flexible tactile sensor has been widely applied to industrial robot and biomedical engineering [1] which requires the flexible tactile sensor have ultra high sensitivity to sense micro-pressure.

Presented flexible tactile sensors can be classified into three types according to the working principle which are capacitive [2-4], piezoelectric [5-7] and piezoresistive [8-13].

Capacitive flexible tactile sensors are using to measure micro-pressure according to the capacitance varied with the compressed elastic material between upper and lower electrodes [2-4]. It has the advantages of high sensitivity, quick response, temperature insensitive and convenience of flexible design [4]. However, the sensitivity of capacitive tactile sensor is fairly ordinary (typical $0.004 \mathrm{kPa}^{-1}$ ) [14] and the parasitic capacitances cased by measuring object is difficult to avoid and eliminate.

Piezoelectric tactile sensors are using to measure micro-pressure according to the piezoelectric effect of flexible piezoelectric materials such as PVDF [5], PZT [6] and ZnO piezoelectric nanowires [7]. 
Piezoelectric tactile sensor has a excellent dynamic performance but ordinary static linearity performance. For example, Witing Liu et al. [5] presented a fingertip piezoelectric tactile sensor array which is working in high frequency $(400 \mathrm{~Hz})$ to measure the surface texture of objects.

Piezoresistive tactile sensors are using to measure the micro-pressure according to piezoresistive effect of flexible resistive materials. Andrea Rinaldi et al. [15] presented a pressure sensor based on Graphene Nanoplatelets. The sensor have a sensitivity of $0.23 \mathrm{kPa}^{-1}$ and a measurement resolution of $1 \mathrm{~Pa}$. However, the sensor have a thickness of $4 \mathrm{~mm}$ which great restrict the application on tactile sensor. Youngdo Jung et al. [16] presented a tactile sensor based on composite material of CNTs/PDMS. The sensor have sensitivity of $0.10173 \mathrm{kPa}^{-1}$ at a range of $0.128-44 \mathrm{kPa}$. However, this sensor have a size of $15 \mathrm{~mm} \times 15 \mathrm{~mm} \times 5 \mathrm{~mm}$ which still have challenge in miniaturization to apply on robot hand.

Therefore, the presented tactile sensors have a high sensitivity but small measurement range or have a large measurement range but low sensitivity. The measurement range and the sensitivity are a pair of contradiction. For example, the presented sensors always have 2-3 orders of magnitude between minimum resolution and maximum measurement range which greatly limited the application in robot hands.

To aiming at the above problem, this paper presented an ultra-sensitivity flexible tactile sensor based on piezoresistive effect of graphene film. The sensor is composed of upper substrate (PDMS), medial film (Graphene/PET) and lower substrate (PI) and is expected to have an ultra high sensitivity and have an excellent characteristic of miniaturization and flexibility. The sensor also performs excellently in sensitivity and measurement range which has 4 orders of magnitude between minimum resolution and maximum measurement range. The sensor may greatly expand the application of flexible tactile sensor in industrial robot and biomedical engineering.

\section{Method}

The structure of the flexible tactile sensor presented in this work is shown as in Figure 1. The sensor was composed of three layers which are upper substrate, medial graphene/PET film and lower substrate. The prepared graphene film was transferred to the PET substrate. A bump was fabricated on the upper substrate to collect and focus stress to the graphene film.

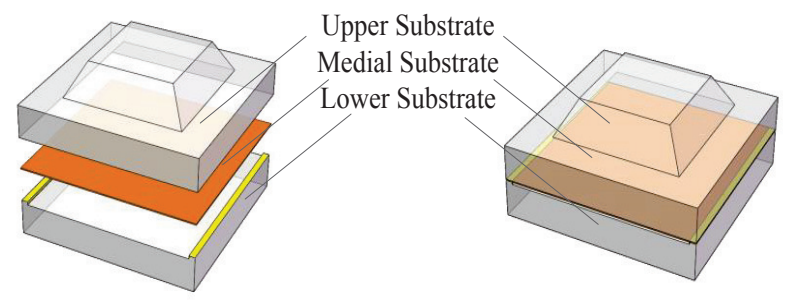

Figure 1. The structure of the flexible tactile sensor.

The principle of the flexible tactile sensor is piezoresistive effect of the graphene/PET film. When a micro-pressure is applied on the surface of the sensor, the upper bump will collect the stress evenly to the graphene film (see Figure 2a,b). These stress will make the C-C bond of the graphene film fractured or cracked (see Figure 2c) and the resistivity of the graphene film changed. Therefore, we can measure the applied micro-pressure according to the varied resistance of the graphene film. Benefiting of the excellent sensitivity and flexibility of graphene film, the sensor will have an ultra-high sensitivity. 


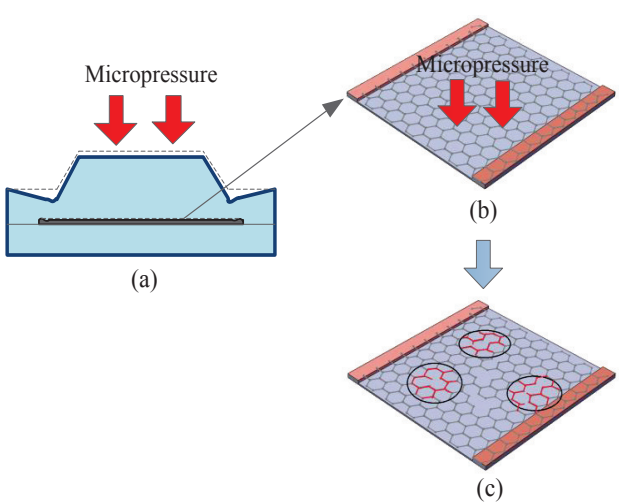

Figure 2. Schematic diagram of working principle of tactile sensor; (a) Schematic diagram of tactile sensor subjected to micro-pressure; (b) Morphology of graphene thin films before being subjected to micro-pressure; (c) Microstructure of graphene film under compression.

\section{Material}

The flexible tactile sensor presented in this work was composed of three layers which is upper substrate, medial graphene film and lower substrate. The upper substrate was made of polydimethylsiloxane (PDMS) which have excellent features of super-elastic, easy processing and chemical stability. The medial sensing film was made of single-layer graphene transferred on Polyethylene terephthalate (PET) film which have features of excellent sensitivity and flexibility. The lower substrate was made of Polyimide (PI) which is flexible and easy to fabricate electrodes.

The upper PDMS substrate was fabricated by a casting method. The specific fabricating process was shown as in Figure 3. Firstly, we fabricated a stainless steel negative mold which is best fitting the size of the upper substrate (see Figure 3a). Secondly, we mixed the silicone elastomer base and elastomer curing of PDMS (SYLGARD 184, Dow Corning Co., Midland, MI, USA) with a rate of 10:1, and put the mixture PDMS into the stainless steel mold (see Figure 3b). Thirdly, we put the stainless steel mold with mixture PDMS into a vacuum drying oven (LiChen, DZF) for $4 \mathrm{~h}$ at a temperature of 80 (see Figure 3c). At last, we peeled off the upper PDMS substrate from the stainless steel mold (see Figure $3 \mathrm{~d}$ ). If the different shape and size of stainless steel mold was prepared, the different shape and size upper PDMS substrate would be fabricated.

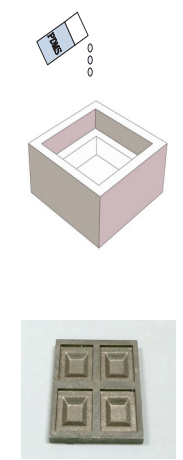

(a)
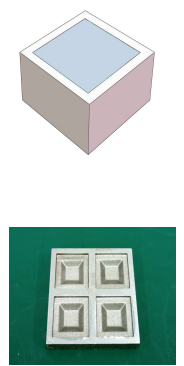

(b)
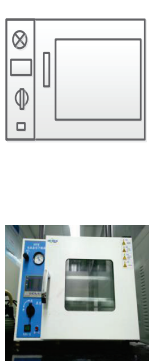

(c)
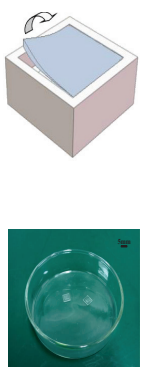

(d)

Figure 3. The fabrication process of upper substrate; (a) A stainless steel negative mold was fabricated; (b) The silicone elastomer base and elastomer curing of PDMS with a rate of 10:1 was fully stirred and then poured into the stainless steel negative mold; (c) Dry the PDMS for $4 \mathrm{~h}$ in a vacuum dryer ar the temperature to $80^{\circ} \mathrm{C}$; (d) Remove the upper substrate from the mold.

The graphene film was fabricated by Chemical vapor deposition (CVD) method and prepared by a wet transfer method. The specific fabrication processing of the flexible tactile sensor was shown as in Figure 4. Firstly, we fabricated a single-layer graphene film on a copper by CVD method 
(see Figure 4a). Secondly, we put the graphene-copper into a solution to etch off copper (see Figure 4c). Then we transferred the graphene on a PET film(see Figure 4d) and pasted them on the PI with the silver, which fabricated the electrodes on a PI(see Figure 4e).At last, we assembled the upper PDMS substrate with the Graphene/PET/FPCB film and the sensor was fabricated (see Figure 4g). The sample devices of the sensor was almost transparent and performed an excellent flexibility with a size of $5 \mathrm{~mm} \times 7 \mathrm{~mm} \times 1 \mathrm{~mm}$ (see Figure 5).

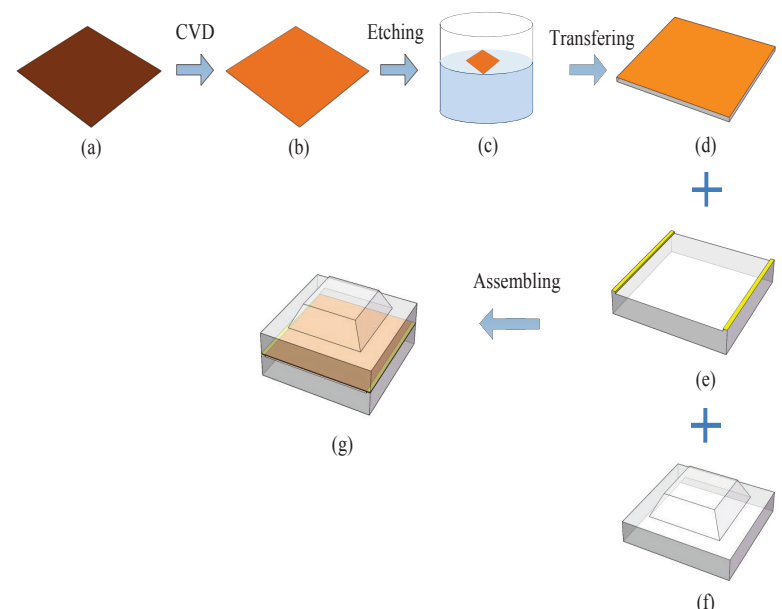

Figure 4. The assembling process of the sensor.

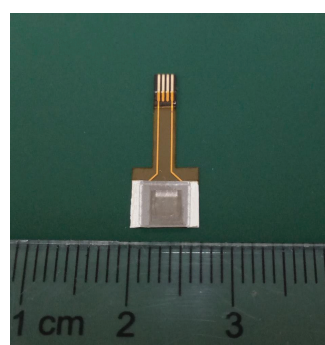

(a)

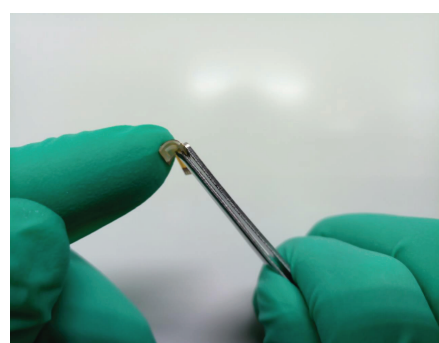

(b)

Figure 5. The photography of the flexible tactile sensor.

\section{Experimental}

To test the performance of the sensor, we designed a micro-pressure testing platform (see Figure 6) which composed of a micro-force gauge (F1128 ZQ-20A-2, ZhiQu Co., Hangzhou, China)and a Lenz Capacitance and Resistance Digital Bridge Measuring Instrument (LCR-8101G, GWINSTEK, Taiwan). The micro-force gauge is able to applied a range of micro-force with a precision of $0.001 \mathrm{~N}$. The LCR Instrument is able to measure and record the output resistance of the sensor with a precision of $0.01 \Omega$. The sample devices of the sensor were placed on the testing bed of the micro-force gauge and connected with the LCR Instrument. When a micro-force was applied on the surface of the sensor by the micro-force gauge, the output resistance of the sensor was displayed and recorded by the LCR instrument.

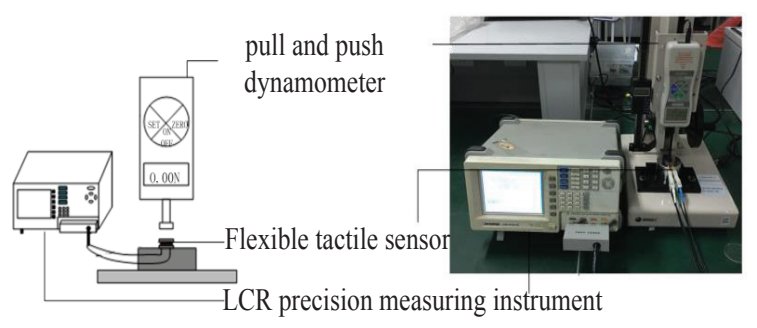

Figure 6. Experiment setup. 


\section{Results and Discussion}

\subsection{Performance}

To validate the working principle of the sensor, we observed the micro-morphology of the sensor by the scanning electron microscope(SEM, JSM-6360LV, Co.JEOL, Tokyo, Japan), see Figure 7a. The micro-morphology of the graphene film of the sensor before pressed and after pressed are shown as in Figure $7 \mathrm{~b}-\mathrm{d}$. Compared with these micro-morphology, we found that the number of crack in the graphene film grew with the increase of pressure applied on the sensor(see Figure $7 \mathrm{c}, \mathrm{d}$ ). The increasing crack will make the resistivity of the graphene film increased and in turns causing the output resistance of the sensor varied with the applied pressure.

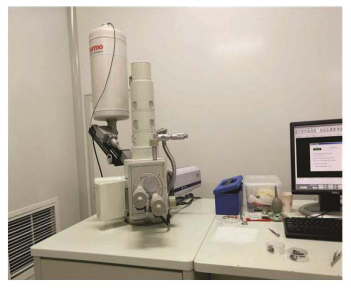

(a)

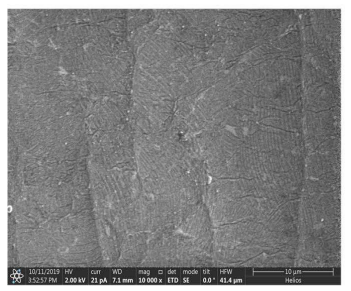

(c)

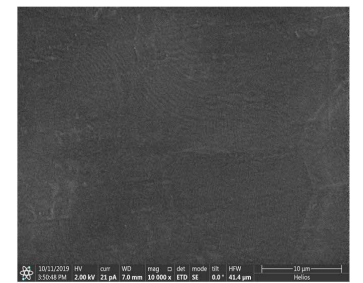

(b)

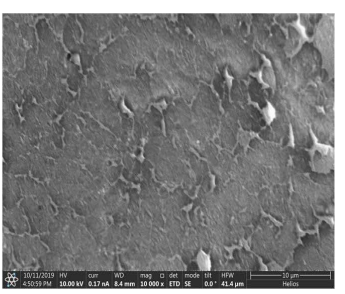

(d)

Figure 7. The sensor was scanned by the scanning electron microscope (a), and the micro-morphology of the graphene film of the sensor before pressed (b), after small pressed (c) and large pressure (d) were observed.

To test the performance of the sensor, we applied the pressure to the sensor and the result is shown as in Figure 8. From the figure we know that the relationship between the output resistance of the sensor and the applied pressure can be fitted by two straight line which are

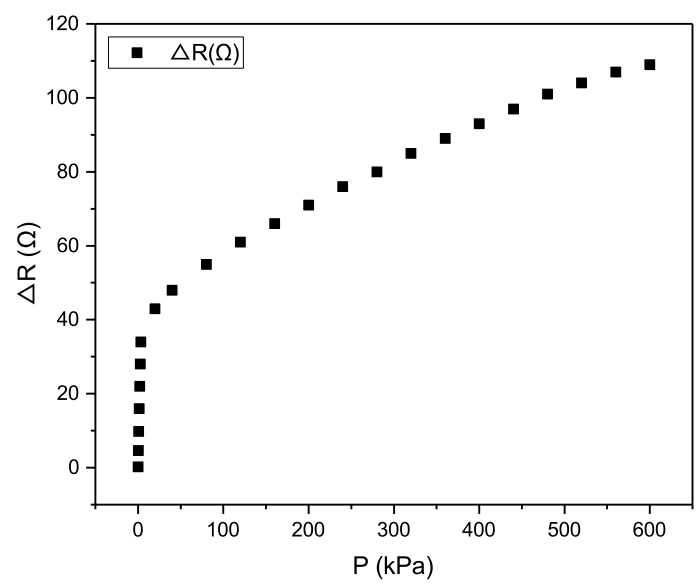

Figure 8. The sensor performed an ultra sensitivity at the range of $0-4 \mathrm{kPa}$ and a large measurement range up to $600 \mathrm{kPa}$. 


$$
\Delta R(p)= \begin{cases}10.56 p+1.24, & p \in[0,4 \mathrm{kPa}] \\ 0.11 p+44.06, & p \in[4 \mathrm{kPa}, 600 \mathrm{kPa}]\end{cases}
$$

From Equation (1), we know that the applied pressure can be measured according to the output resistance of the sensor. The sensor is able to measure a large range of $0-600 \mathrm{kPa}$ pressure with a high sensitivity.

\subsection{Sensitivity}

Sensitivity is the ratio of the increment of the output to the increment of the input when the measurement system is performing a static measurement, which is

$$
S=\lim _{\Delta x \rightarrow 0}\left(\frac{\Delta y}{\Delta x}\right)=\frac{d_{y}}{d_{x}}
$$

For a linear measurement system, the sensitivity is

$$
S=\frac{y}{x}=K=\frac{m_{y}}{m_{x}} \tan \theta
$$

Where $m_{x}$ and $m_{y}$ are the scale of the $x$-axis and $y$-axis respectively. $\theta$ is the angle between the tangent of the corresponding point and the $x$-axis. In other words, the sensitivity of the linear measurement system is a constant and can be obtained from the slope of the static characteristic curve.

In this work, we use the relative varies of the output resistance $(\Delta R)$ as the $y$-axis and the applied pressure $(\mathrm{P})$ as the $x$-axis of the static characteristic curve, i.e., $\Delta R$-P curve. Therefore, the sensitivity of the sensor can be written by

$$
S=\frac{\Delta R}{P}
$$

Where $\Delta R$ is the output varies of the sensor (the unit is $\Omega$ ). $P$ is the applied pressure (the unit is $\mathrm{kPa}$ ). Therefore, the unit of the sensitivity is $\Omega / \mathrm{kPa}$. From Equation (4), we know that the output resistance varies from 0 to $42 \Omega$ at the range of $0-4 \mathrm{kPa}$, therefore, we obtain that

$$
S_{1}=\frac{41.8}{4-0.004}=10.56 \Omega / \mathrm{kPa}
$$

The sensitivity of the sensor at the range of $4-600 \mathrm{kPa}$ is

$$
S_{2}=\frac{|109-41.8|}{600-4}=0.113 \Omega / k P a
$$

Therefore, we concluded that the sensor have a high sensitivity of $10.56 \Omega / \mathrm{kPa}$ at the range of $\sim 4 \mathrm{kPa}$ and a sensitivity of $0.113 \Omega / \mathrm{kPa}$ at the range of $4-6 \mathrm{kPa}$.

\subsection{Linearity}

Linearity is the degree of deviation between the actual input-output relationship and the fitting input-output relationship of a sensor. It is usually expressed by the maximum nonlinear error, which is

$$
\delta_{L}=\frac{\Delta L_{\max }}{Y_{F S}} \times 100 \%
$$

Where $\delta_{L}$ is the linearity; $\Delta L_{\max }$ is the maximum deviation between the calibration line and the fitted line; $Y_{F S}$ is the full scale of the measurement range. In this work, we used the least squares method to fit the line. Therefore, the linearity refers to the least squares linearity. 
From Figure 9a we obtain that the linearity of the sensor presented in this work at the range of $0-4 \mathrm{kPa}$ can be given by

$$
\delta_{L 1}=\frac{|21.2-19.8|}{42.6} \times 100 \%=3.28 \%
$$

The linearity of the sensor presented in this work at the range of 4-600 kPa can be given by

$$
\delta_{L 2}=\frac{|72.5-67.7|}{115} \times 100 \%=4.17 \%
$$

From Equations (8) and (9) we know that the sensor performed a better linearity at the low range of less than $4 \mathrm{kPa}$ and a well linearity at the large range up to $600 \mathrm{kPa}$. These two linear input-output curve makes the sensor has an ultra-high sensitivity as well as has a large measurement range.

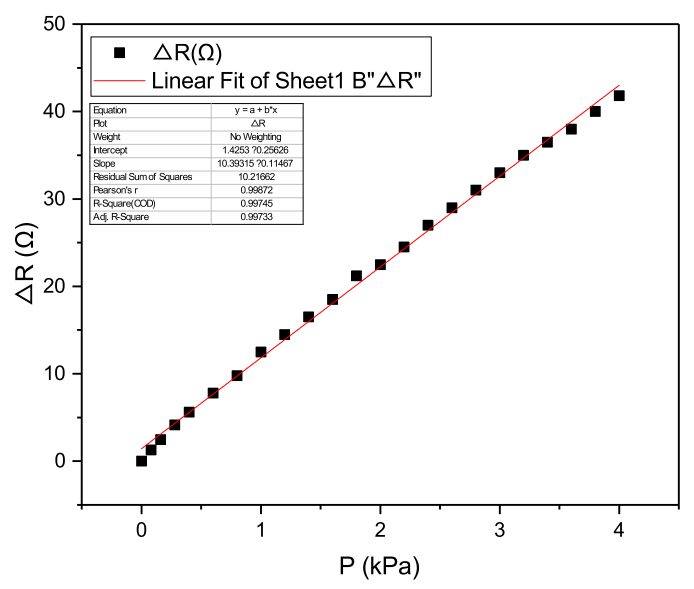

(a)

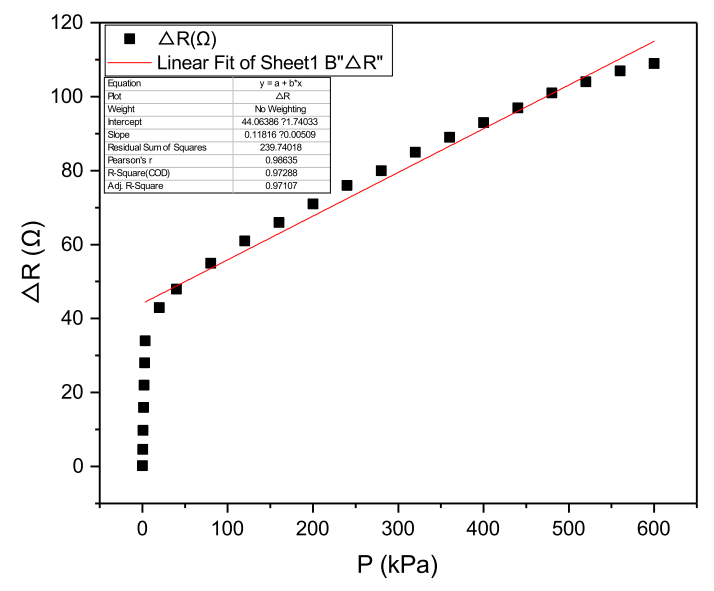

(b)

Figure 9. The linearity of the sensor is $3.28 \%$ at the range of $0-4 \mathrm{kPa}(\mathbf{a})$ and $4.17 \%$ at the range of $4-600 \mathrm{kPa}(\mathbf{b})$.

\subsection{Hysteresis}

Hysteresis refers to the phenomenon in which the input-output curve of the sensor is not coincident between the positive stroke and negitive stroke. It usually expressed by the maximum hysteresis error, which is

$$
\delta_{H}=\frac{\left|\Delta H_{\max }\right|}{Y_{F S}} \times 100 \%
$$


Where $\delta_{H}$ is the maximum hysteresis error; $\Delta H_{\text {max }}$ is the maximum deviation between the positive and the negative stroke; $Y_{F S}$ is the full scale of the measurement range.

In this work, the hysteresis curve of the sensor is shown as in Figure 10a. According to Figure 10a, we obtain that the hysteresis error of the sensor at the range of $0-4 \mathrm{kPa}$ can be given by

$$
\delta_{H}=\frac{|39.5-37|}{42.6} \times 100 \%=5.87 \%
$$

The hysteresis error of the sensor at the range of 4-600 kPa can be given by

$$
\delta_{H}=\frac{|44-40|}{115-42.6} \times 100 \%=5.52 \%
$$

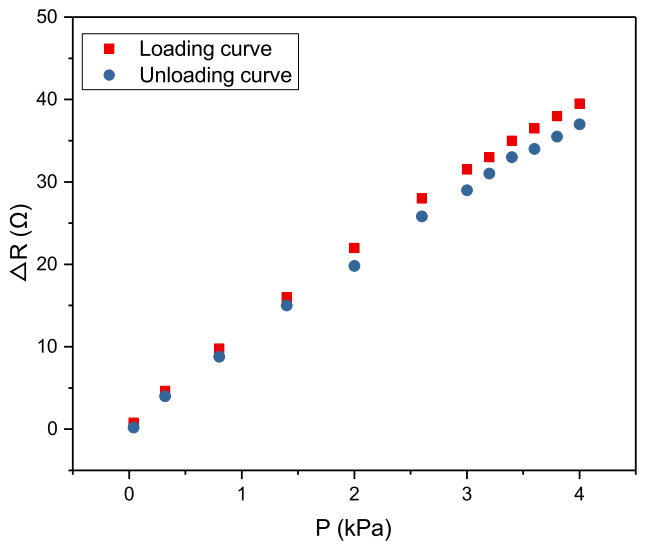

(a)

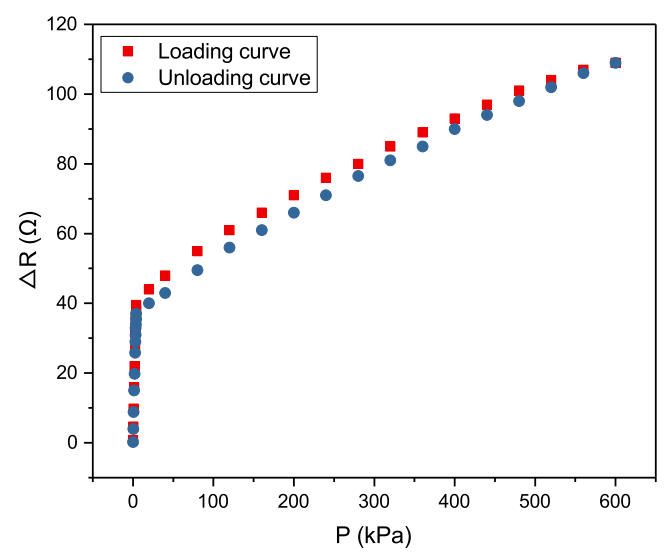

(b)

Figure 10. The hysteresis of the sensor is $5.87 \%$ at the range of $0-4 \mathrm{kPa}$ (a) and $5.52 \%$ at the range of $4-600 \mathrm{kPa}(\mathbf{b})$.

\subsection{Repeatability}

Repeatability is the inconsistency of the characteristic curves when the same sensor is tested under the same working conditions for multiple executions of the experiment. It is expressed by repeatability error which is given by

$$
\delta_{R}=\frac{\left|Z \delta_{\max }\right|}{Y_{F S}} \times 100 \%
$$


where $\delta_{R}$ is the repeatability error. $Z$ is the confidence coefficient. For the normal distribution, the confidence probability is $99.73 \%$ when $Z$ equals $3 . \delta_{\max }$ is the maximum value of the standard deviation of each measurement point. $Y_{F S}$ is the full-scale of the sensor.

The standard deviation $\delta_{\max }$ can be calculated by the Bessel Formula, which is

$$
\delta_{\max }=\max (\delta)=\sqrt{\frac{1}{N-1} \sum_{i=1}^{N}\left(R_{i}-\bar{R}\right)^{2}}
$$

where $i$ is the index of the measurement point; $R_{i}$ is the corresponding measurement and $\bar{R}$ is the average value of the measurement points.

In this work, the hysteresis curve of the sensor is shown as in Figure 11. According to the figure, we obtain that the hysteresis of the sensor at the range of $0-4 \mathrm{kPa}$ can be given by

$$
\delta_{H_{1}}=\frac{\Delta H_{\max }}{Y_{F S}} \times 100 \%=\frac{|39.5-37|}{42.6} \times 100 \%=5.87 \%
$$

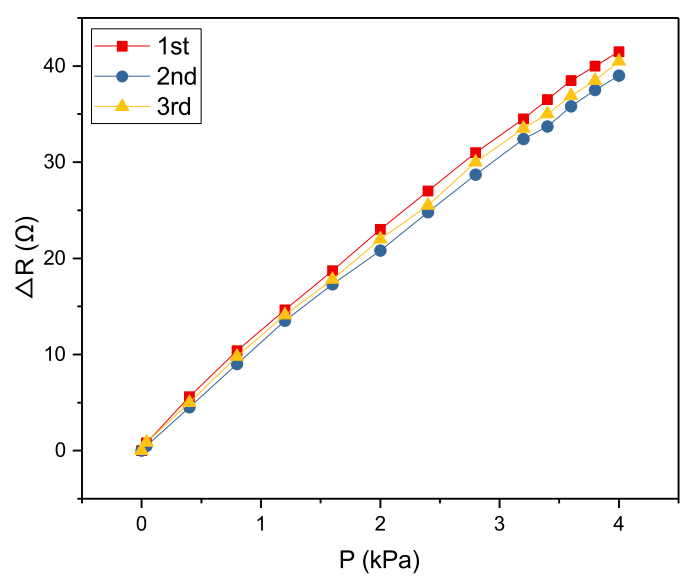

(a)

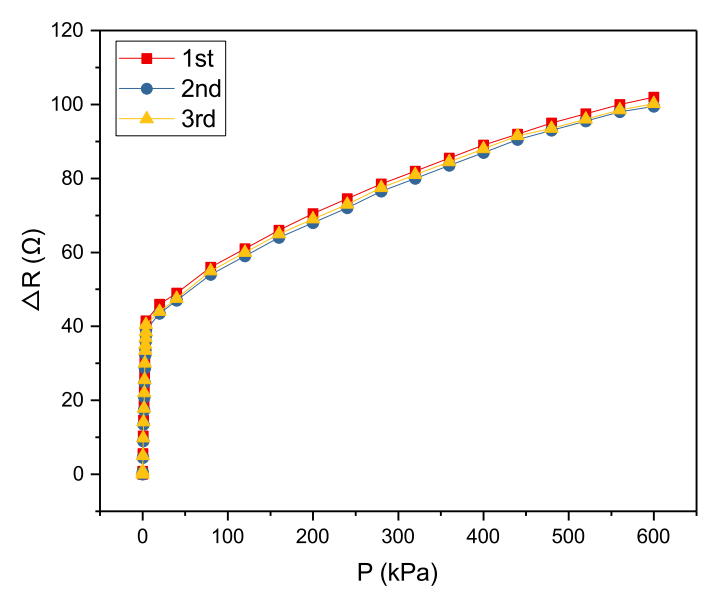

(b)

Figure 11. The repeatability of the sensor is $5.87 \%$ at the range of $0-4 \mathrm{kPa}(\mathrm{a})$ and $5.52 \%$ at the range of $4-600 \mathrm{kPa}(\mathbf{b})$. 
The hysteresis of the sensor at the range of 4-600 $\mathrm{kPa}$ can be given by

$$
\delta_{H_{2}}=\frac{\Delta H_{\max }}{Y_{F S}} \times 100 \%=\frac{|44-40|}{115-42.6} \times 100 \%=5.52 \%
$$

The cycling test of the fabricated tactile sensor is carried out by loading a force of $200 \mathrm{kPa}$. Results showed that the sensor has good repeatability during 500 cycles, as shown in Figure 12.

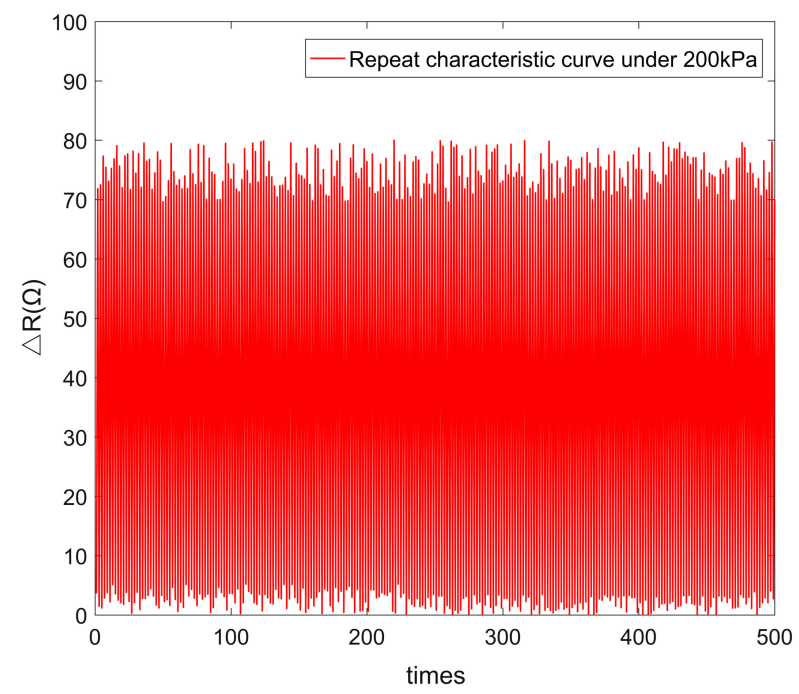

Figure 12. The cycling test of the tactile sensor applied with a force of $200 \mathrm{kPa}$.

\subsection{Dynamic Performance}

The dynamic characteristic refers to the response time after the sensor's input changes. The response time is the delay time between the output and corresponding input. For a tactile sensor, the dynamic performance refers to the load and unload times of the sensor.

In this work, we load a force of $0.02 \mathrm{~N}$ to the sensor repeatability and record the output capacitance of the sensor by a source meter(Keithley 2450, Tektronix, Beaverton, OR, USA) with a sampling period of $10 \mathrm{~ms}$. The dynamic characteristic of the sensor is shown as in Figure 13. From the figure, we obtain that the loading time and the unloading time of the sensor is $10 \mathrm{~ms}$ and $30 \mathrm{~ms}$, respectively.

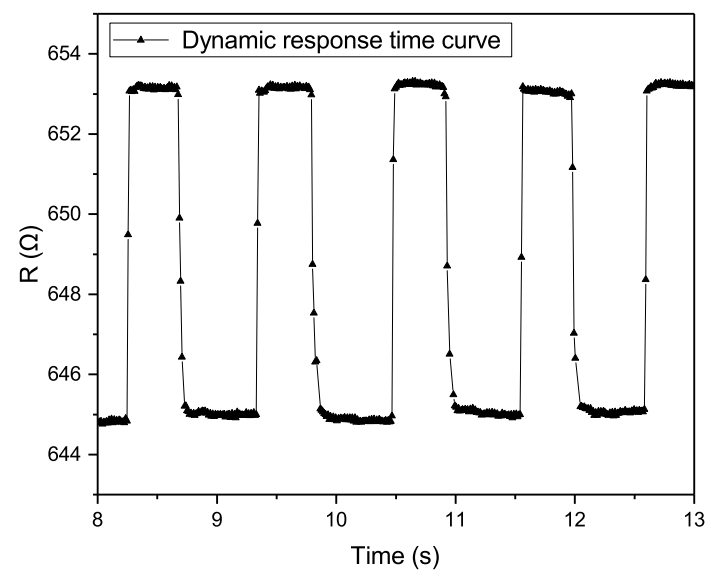

Figure 13. The sensor is based on a PI substrate and four Ni thermal element. 


\subsection{Flexibility}

The flexible property of fabricated tactile sensor are tested on the curved surface with radius of curvature from $10 \mathrm{~mm}$ to $30 \mathrm{~mm}$. Results showed that the tactile sensor features good flexibility and repeatability with various radius of curvature. As shown in Figure 14a, with the decrease of the radius of curvature of the curved surface, the initial resistance of the sensor mounted reduces slowly. Therefore, the proposed flexible sensor can be utilized for fingertip of robotic dexterous hand to provide haptic perception, as shown in Figure 14b.

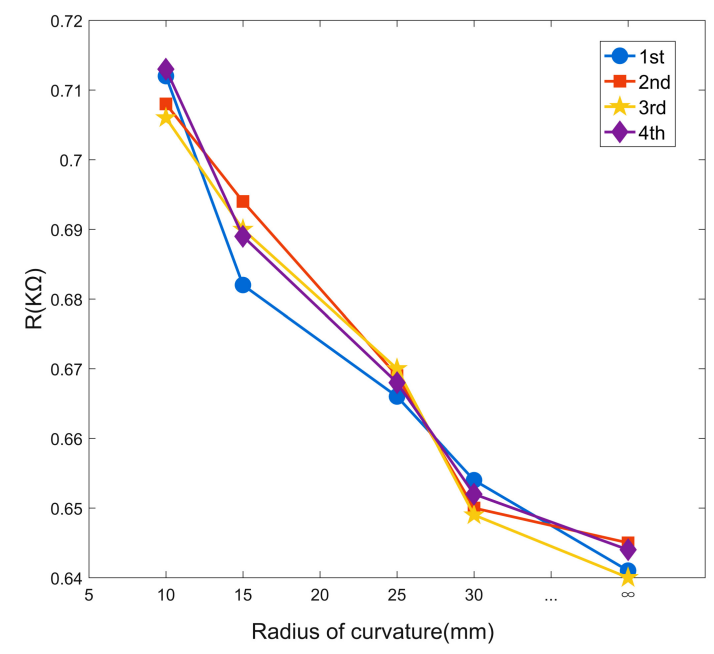

(a)

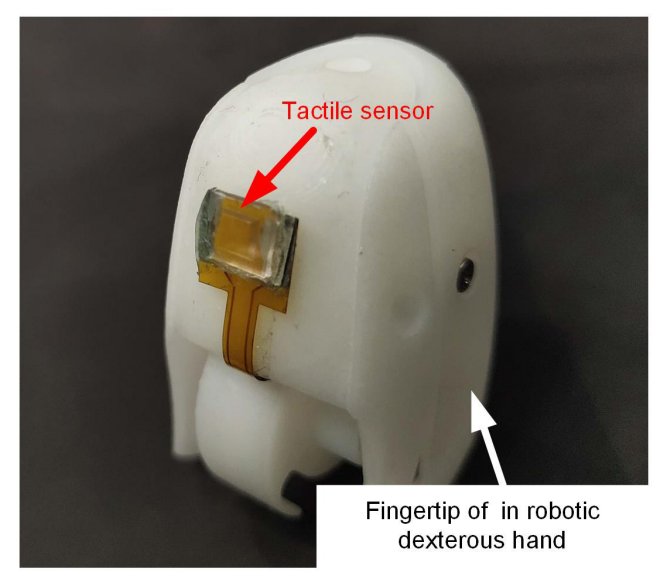

(b)

Figure 14. The flexility of the sensor: (a) cycling test under force of $200 \mathrm{kPa}$ and (b) mounted on the fingertip of robotic dexterous hand.

\subsection{Sensors Comparisone}

To compare our work with the state of the art, we given a table to list the material, sensitivity, precision, measurement range, span scale, response time (RT) and the size of the references sensors (see Table 1). The span scale is the ratio between the minimum resolution and maximum measurement range of the sensor. For a tactile sensor, a better span scale means the sensor has a good minimum resolution as well as has a large measurement range. In this work, the span scale of our presented sensor equals 4 , which means there are 4 orders magnitude between the minimum resolution and maximum measurement range. This result increased by 1-2 order of magnitude compared with other refereces (2-3 orders of magnitudes). From the table, we also know that the sensor of our work 
have excellent performance in precision $(0.004 \mathrm{kPa})$, large measurement range $(600 \mathrm{kPa})$, high response time $(10 \mathrm{~ms})$ and small size $(5 \mathrm{~mm} \times 7 \mathrm{~mm})$.

Table 1. Sensors comparison.

\begin{tabular}{cccccccc}
\hline Ref. & Material & $\begin{array}{c}\text { Sensitivity } \\
\left(\mathbf{k P a}^{-\mathbf{1}} \mathbf{)}\right.\end{array}$ & $\begin{array}{c}\text { Precision } \\
\mathbf{( k P a )}\end{array}$ & $\begin{array}{c}\text { Measuring Range } \\
\mathbf{( k P a})\end{array}$ & Span Scale & $\begin{array}{c}\text { RT } \\
(\mathbf{m s})\end{array}$ & $\begin{array}{c}\text { Size } \\
(\mathbf{m m})\end{array}$ \\
\hline$[12]$ & $\mathrm{AgNWs} / \mathrm{rGO}$ & 5.8 & 0.000125 & $0-0.1$ & 2 & 29.5 & $20 \times 1 \times 1$ \\
\hline$[16]$ & $\mathrm{CNT} / \mathrm{PDMS}$ & 0.0173 & 0.128 & $0.128-44$ & 2 & - & $15 \times 15 \times 5$ \\
\hline$[17]$ & $\mathrm{ITO} / \mathrm{SSNPs}-\mathrm{PU} / \mathrm{ITO}$ & 2.46 & 0.3 & $0.3-24.5$ & 1 & 30 & - \\
\hline$[18]$ & $\mathrm{rGO}$ & -5.53 & 0.0015 & $0-1$ & 2 & 0.2 & $>100 \times 100$ \\
\hline$[19]$ & $\mathrm{CNTs} / \mathrm{CB} / \mathrm{SR}$ & $>0.04$ & $0.1 \mathrm{~N}$ & $0-1562.5(0-100 \mathrm{~N})$ & 3 & - & $\mathrm{R}=4$ \\
\hline $\begin{array}{c}\text { Our } \\
\text { work }\end{array}$ & $\mathrm{GR}$ & 0.04 & 0.004 & $0-600$ & 4 & 10 & $5 \times 5 \times 5$ \\
\hline
\end{tabular}

\section{Conclusions}

In this work, we presented a ultra-sensitivity flexible tactile sensor based on graphene film. The sensor is based on piezoresistive effect of graphene film and is composed of upper substrate (PDMS with a thickness of $5 \mu \mathrm{m}$ and a bump thickness of $1 \mathrm{~mm}$ ), medial graphene film (graphene film with a size of $5 \mathrm{~mm} \times 7 \mathrm{~mm}$ ) and lower substrate (PET with a hardness of $2 \mathrm{H}$ ). We fabricated sample sensor devices and carried out experiment to test the performance. According to the result, we concluded that the sensor is able to measure the pressure at the range of $0-600 \mathrm{kPa}$. The sensor has an ultra-sensitivity of $10.56 \Omega / \mathrm{kPa}$ at the range of $0-4 \mathrm{kPa}$ with a linearity error, hysteresis error and repeatability of $3.28 \%, 5.87 \%$ and $4.92 \%$ respectively. The sensor has a sensitivity of $0.113 \Omega / \mathrm{kPa}$ at the range of 4-600 kPa with a linearity error, hysteresis error and repeatability of $4.17 \%, 5.52 \%$ and $4.79 \%$ respectively. The sensor performs an excellent dynamic characteristic of loading time of $10 \mathrm{~ms}$ and unloading time of $30 \mathrm{~ms}$. The sensor also has 4 orders of magnitude between minimum resolution and maximum measurement range which have great advantage compared with state of the art. The sensor is expected have great application prospect in robot and biomedical.

Author Contributions: Conceptualization, X.L.; Data curation, H.H.; Formal analysis, L.Q.; Funding acquisition, G.B. and J.C.; Project administration, X.L.; Supervision, X.L. and W.B.; Writing - original draft, L.Q.

Funding: The authors thank the funding of the National Natural Science Foundation of China (Grant No. 61601342), the Natural Science Basic Research Plan in Shaanxi Province of China (Program No. 2016ZDJC-07) and the Shaanxi Postdoctoral Science Foundation funded project (Grant No. 2017BSHYDZZ36).

Conflicts of Interest: The authors declare no conflict of interest.

\section{References}

1. Novoselov, K.S. ; Geim, A.K. ; Morozov, S.V. ; Jiang, D.; Zhang, Y. ; Dubonos, S.V. ; Grigorieva, I.V.; Firsov, A.A. Electric field effect in atomically thin carbon films. Science 2004, 306, 666-669. [CrossRef] [PubMed]

2. Huang, Y.; Yuan, H.; Kan, W.; Guo, X.; Liu, C.; Liu, P. A fexible three-axial capacitive tactile sensor with multilayered dielectric for artifcial skin applications. Microsyst. Technol. 2017, 23, 1847-1852. [CrossRef]

3. Chen, L.; Liu, J.; Wang, X.; Ji, B.; Chen, X.; Yang, B. Flexible Capacitive Hydrogel Tactile Sensor With Adjustable Measurement Range Using Liquid Crystal and Carbon Nanotubes Composites. IEEE Trans. Electron Devices 2017, 64, 1968-1972. [CrossRef]

4. Wan, S.; Bi, H.; Zhou, Y.; Xie, X.; Su, S.; Yin, K.; Sun, L. Graphene oxide as high-performance dielectric materials for capacitive pressure sensors. Carbon 2017, 114, 209-216. [CrossRef]

5. Yu, P.; Liu, W.; Gu, C.; Cheng, X.; Fu, X. Flexible Piezoelectric Tactile Sensor Array for Dynamic Three-Axis Force Measureme. Sensors 2016, 16, 819. [CrossRef] [PubMed]

6. Yu, P.; Liu, W.; Gu, C.; Cheng, X.; Fu, X. Fingertip Piezoelectric Tactile Sensor Array for Roughness Encoding Under Varying Scanning Velocity. IEEE Sens. J. 2017, 17, 6867-6879. 
7. Sim, M.; Jeong, Y.; Lee, K.; Shin, K.; Park, H.; Sohn, J. I.; Cha, S.N.; Jang, J.E. Psychological tactile sensor structure based on piezoelectric sensor arrays. RSC Adv. 2015, 5, 40363-40368.

8. Hoang, P.T.; Phung, H.; Nguyen, C.T.; Nguyen, T.D.; Choi, H.R. A Highly Flexible, Stretchable and Ultra-thin Piezoresistive Tactile Sensor Array using PAM/PEDOT:PSS Hydrogel. In Proceedings of the International Conference on Ubiquitous Robots and Ambient Intelligence (URAI), Jeju, South Korea, 28 June-1 July 2017; pp. 950-955.

9. Shi, J.; Hu, J.; Dai, Z.; Zhao, W.; Liu, P.; Zhao, L.; Guo, Y.; Yang, T.; Zou, L.; Li, H.; et al. Graphene welded carbon nanotube crossbars for biaxial strain sensors. Carbon 2017, 123, 786-793. [CrossRef]

10. Luo, Y.; Xiao, Q.; Li, B. A Stretchable Pressure-Sensitive Array Based on Polymer Matrix. Sensors 2017, 123, 786-793.

11. Huang, Y.; He, X.; Gao, L.; Wang, Y.; Liu, C.; Liu, P. Pressure-sensitive carbon black graphene nanoplatelets silicone rubber hybrid conductive composites based on a three-dimensional polydopamine-modified polyurethane sponge. J. Mater. Sci. Mater. Electron. 2017, 28, 9495-9504. [CrossRef]

12. Wei, Y.; Chen, S.; Dong, X.; Lin, Y.; Liu, L. Flexible piezoresistive sensors based on dynamic bridging effect of silver nanowires toward graphene. Carbon 2017, 113, 395-403. [CrossRef]

13. Xu, M.; Qi, J.; Li, F.; Liao, X.; Liu, S.; Zhang, Y. Ultra-thin, transparent and flexible tactile sensors based on graphene films with excellent anti-interference. RSC Adv. 2017, 7, 30506-30512. [CrossRef]

14. Lipomi, D.J.; Vosgueritchian, M.; Tee, B.C.; Hellstrom, S.L.; Lee, J.A.; Fox, C.H.; Bao, Z. Skin-like pressure and strain sensors based on transparentelastic films of carbon nanotubes. Nat. Nanotechnol. 2011, 6, 788-792. [CrossRef] [PubMed]

15. Rinaldi, A.; Tamburrano, A.; Fortunato, M.; Sarto, M. A Flexible and Highly Sensitive Pressure Sensor Based on a PDMS Foam Coated with Graphene Nanoplatelets. Sensors 2016, 16, 2148. [CrossRef] [PubMed]

16. Jung, Y.; Lee, D.G.; Park, J.; Ko, H.; Lim, H. Piezoresistive Tactile Sensor Discriminating Multidirectional Forces. Sensors 2015, 15, 25463-25473. [CrossRef] [PubMed]

17. Lee, D.; Lee, H.; Jeong, Y.; Ahn, Y.; Nam, G.; Lee, Y. Highly Sensitive, Transparent, and Durable Pressure Sensors Based on Sea-Urchin ShapedMetal Nanoparticles. Adv. Mater. 2016, 28, 9364-9369. [CrossRef] [PubMed]

18. Zhu, B.; Niu, Z.; Wang, H.; Leow, W.R.; Wang, H.; Li, Y.; Zheng, L.; Wei, J.; Huo, F.; Chen, X. Microstructured Graphene Arrays for Highly Sensitive Flexible Tactile Sensors. Small 2014, 10, 3625-3631. [CrossRef] [PubMed]

19. Huang, Y.; Fang, D.; Wu, C.; Wang, W.; Guo, X.; Liu, P. A flexible touch-pressure sensor array withwireless transmission system for robotic skin. Rev. Svi. Instrum. 2017, 87, 065007. [CrossRef] [PubMed]

Sample Availability: Samples of the compounds are available from the authors.

(C) 2019 by the authors. Licensee MDPI, Basel, Switzerland. This article is an open access article distributed under the terms and conditions of the Creative Commons Attribution (CC BY) license (http://creativecommons.org/licenses/by/4.0/). 